\title{
Semi-Markov model of a technical system with calendar maintenance
}

\author{
Sergey G. Glech ${ }^{1}$, Yuriy E. Obzherin ${ }^{2, *}$, and Mikhail M. Nikitin ${ }^{2}$ \\ ${ }^{1}$ Sevastopol Institute of Economics and Humanities, Department of Management, Sevastopol, Russia \\ ${ }^{2}$ Sevastopol State University, Higher Mathematics Department, Sevastopol, Russia
}

\begin{abstract}
Maintenance is a widely used method of reliability and efficiency increasing of technical systems. In present paper, a semi-Markov model of the system with calendar maintenance was constructed. Phase merging algorithm is used for approximate finding of system's stationary reliability characteristics. The problem of optimum maintenance carryingout period finding is solving.
\end{abstract}

\section{Introduction}

Maintenance is an important method of technical systems reliability and efficiency increasing [1-5]. Maintenance is understood as an operation or a set of operations to maintain the operability or serviceability of the product when used for its intended purpose, storage and transportation.

Maintenance is especially important in automated production, where productivity, unlike mechanized production, is high enough, and failures lead to significant economic losses. It is necessary to plan a maintenance service aimed at preventing failures, and, consequently, there is a need to develop mathematical models of technical systems taking the maintenance into account.

Herewith maintenance periodicity optimization is one of the actual problems; the reliability theory is required to solve it [6]. Semi-Markov processes are often used for construction of systems' models for various purposes [7 -9].

In this paper a semi-Markov model with a discrete-continuous phase space of states of a technical system was constructed, taking into account the calendar maintenance.

The algorithm of phase merging, developed in the works of V.S. Korolyuk, A.F. Turbin, A.V. Swishchuk, is used for approximate finding of systems reliability characteristics [9 11]. The results obtained were used to find the optimal period of maintenance.

\section{Description of the system}

Let us consider the system functioning. Time to failure of the system is random variable (RV) $\alpha_{1}$ with distribution function (DF) $F_{1}(t)=P\left(\alpha_{1} \leq t\right)$, renewal time is RV $\beta_{1}$ with DF $G_{1}(t)=P\left(\beta_{1} \leq t\right)$. At random time moments (with time intervals $\alpha_{2}$ with DF

\footnotetext{
*Correspondent author: objsev@mail.ru
} 
$\left.F_{2}(t)=P\left(\alpha_{2} \leq t\right)\right)$ a maintenance is carried out, maintenance time is RV $\beta_{2}$ with DF $G_{2}(t)=P\left(\beta_{2} \leq t\right)$. Maintenance is carried out if its initial moment is within the period of system operation. After the maintenance is carried out the system operation starts from the beginning (the operation properties of the system are being completely renewed). RV $\alpha_{1}$, $\alpha_{2}, \beta_{1}, \beta_{2}$ are supposed independent and having finite expectations and dispersions, moreover DF $F_{1}(t), G_{1}(t), G_{2}(t)$ have distribution densities (DD) $f_{1}(t), g_{1}(t), g_{2}(t)$.

\section{Construction of a semi-Markov model of the system and its characteristics determination}

To describe system functioning let us use semi-Markov process $\xi(t)$ with the next set of states

$$
E=\{221,210,102 x, 112 x, 200 x\}
$$

where

221 - the maintenance is begun, the system operation is interrupted;

210 - the maintenance is finished, the system operation is started from the beginning;

$102 x$ - the system has finished its operation, the renewal is begun, the time to maintenance is $\mathrm{x}>0$;

$112 x$ - the renewal of the system has taken place, the time to the next maintenance is $x>0$; $200 x$ - the maintenance is not carried out because the moment of its beginning is within system renewal, time to renewal finishing is $x>0$.

Time diagram of system functioning is shown in Fig. 1.

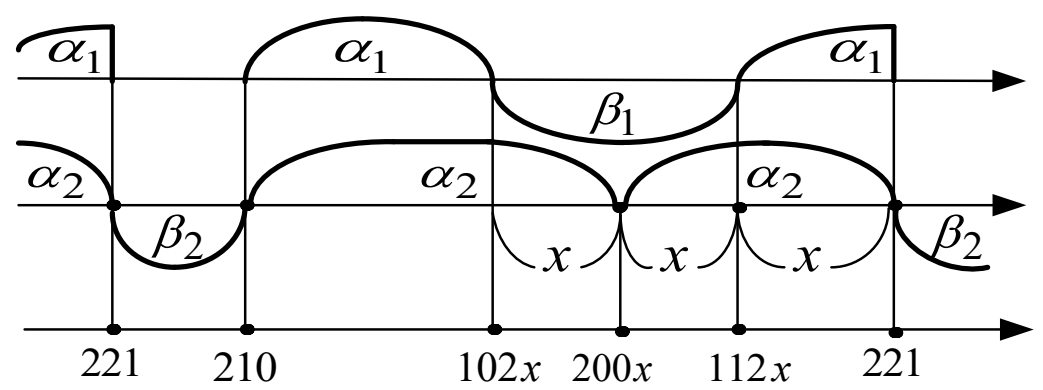

Fig. 1. Time diagram of system functioning.

Let us proceed to the finding of the system's reliability stationary characteristics. Let us use an approximate method of stationary characteristics obtaining based on phase merging algorithm [10-12].

Suppose that embedded Markov chain (EMC) $\left\{\xi_{n} ; n \geq 0\right\}$ kernel for the semi-Markov process $\xi(t)$ is close to the EMC $\left\{\xi_{n}^{(0)} ; n \geq 0\right\}$ kernel for the supporting system $S_{0}$ with single stationary distribution $\rho(d x)$.

Then the next approximate formulas [9] can be used for approximate calculation of the average stationary time to failure $T_{+}$, the average stationary renewal time $T_{-}$and the stationary availability factor $K_{a}$ of the real system $\mathrm{S}$ : 


$$
T_{+} \approx \frac{\int_{E_{+}} m(x) \rho(d x)}{\int_{E_{+}} \rho(d x) P\left(x, E_{-}\right)}, \quad T_{-} \approx \frac{\int_{E_{-}} m(x) \rho(d x)}{\int_{E_{-}} \rho(d x) P\left(x, E_{+}\right)}, \quad K_{a}=\frac{T_{+}}{T_{+}+T_{-}}
$$

where $m(x)$ are the average sojourn times in state $x \in E$ of the initial system; $P(x, d y)$ are the transition probabilities for EMC $\left\{\xi_{n} ; n \geq 0\right\}$ of the initial system.

Let us choose a supporting system $S_{0}$. Suppose that the real system's time to failure is significantly longer than the renewal time and the time of the maintenance. This leads to the fact that the supporting system $S_{0}$ is a system that is recovered instantly and has instant maintenance. Time diagram of the supporting system $S_{0}$ functioning is represented in Fig.2.

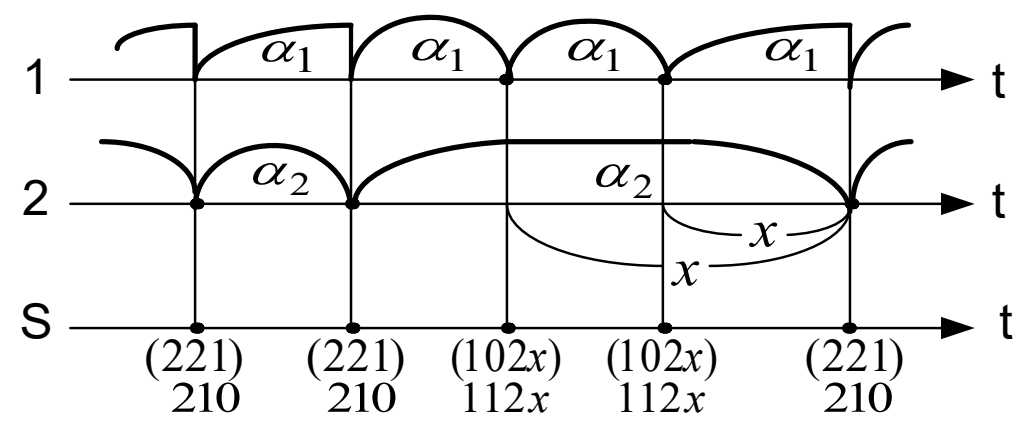

Fig. 2. Time diagram of the supporting system $S_{0}$ functioning.

Let us define transitions probabilities of embedded Markov chain (EMC) $\left\{\xi_{n}^{0} ; n \geq 0\right\}$ for supporting system.

$$
\begin{gathered}
P_{210}^{221}=\int_{0}^{\infty} \bar{F}_{1}(y) d F_{2}(y) ; \quad p_{210}^{102 x}=\int_{0}^{\infty} f_{1}(y) d_{y} F_{2}(x+y) ; P_{221}^{210}=1 ; \\
p_{112 x}^{102 y}=f_{1}(x-y), 0<y<x ; P_{102 x}^{112 x}=1 ; P_{112 x}^{221}=\bar{F}_{1}(x) .
\end{gathered}
$$

Let us denote by $\rho_{0}, \rho_{1}$ the values of EMC stationary distribution $\left\{\xi_{n}^{0} ; n \geq 0\right\}$ on states 210,211 respectively and assume the existence of stationary densities $\rho_{102}(x), \rho_{112}(x)$ for states $102 x, 112 x$ respectively. Let us construct a system of integral equations for them:

$$
\left\{\begin{array}{l}
\rho_{1}=\rho_{0} \int_{0}^{\infty} \bar{F}_{1}(y) d F_{2}(y)+\int_{0}^{\infty} \bar{F}_{1}(y) \rho_{112}(y) d y ; \rho_{0}=\rho_{1} ; \rho_{112}(x)=\rho_{102}(x) \\
\rho_{102}(x)=\rho_{0} \int_{0}^{\infty} f_{1}(y) d_{y} F_{2}(x+y)+\int_{x}^{\infty} f_{1}(y-x) \rho_{112}(y) d y, x>0 \\
\rho_{0}+\rho_{1}+\int_{0}^{\infty} \rho_{102}(x) d x+\int_{0}^{\infty} \rho_{112}(x) d x=1
\end{array}\right.
$$

the last equation of the system is a normalization requirement.

The system of equations (2) has the following solution: 


$$
\rho_{112}(x)=\rho_{0} \int_{0}^{\infty} h_{1}(y) d_{y} F_{2}(x+y)
$$

where $h_{1}(y)=\sum_{n=1}^{\infty} f_{1}^{*(n)}(y)$ is the density of renewal function, the constant $\rho_{0}$ is obtained in an explicit form from the normalization requirement.

The class of ergodic states of the supporting system $S_{0}$ is

$$
E^{0}=\{210,221,102 x, 112 x\}
$$

For initial system $S$ the sets of up-states $E_{+}$and down-states $E_{\text {. are }}$

$$
E_{+}=\{210,112 x\}, E_{-}=\{221,200 x, 102 x\} .
$$

Let us proceed to the finding of the initial system's stationary characteristics. Let us define the EMC $\left\{\xi_{n}, n \geq 0\right\}$ transition probabilities for the initial system.

$$
\begin{gathered}
p_{200 x}^{200 d y}=F_{2}(x-d y), \quad 0<y<x, \quad p_{200 x}^{112 d y}=F_{2}(x+d y), \quad y>0 ; \\
p_{112 x}^{102 y}=f_{1}(x-y), 0<y<x, P_{112 x}^{211}=\bar{F}_{1}(x) ; p_{102 x}^{112 y}=g_{1}(x-y), 0<y<x, \\
p_{102 x}^{200 y}=g_{1}(x+y), y>0 ; P_{221}^{210}=1 ; \\
P_{210}^{221}=\int_{0}^{\infty} \bar{F}_{1}(y) d F_{2}(y), p_{210}^{102 x}=\int_{0}^{\infty} f_{1}(y) d_{y} F_{2}(x+y) .
\end{gathered}
$$

Let us find the average values of the sojourn times in the states of the initial system, which are represented by the formulas:

$$
\theta_{221}=\beta_{2}, \theta_{210}=\alpha_{1} \wedge \alpha_{2}, \theta_{112 x}=\alpha_{1} \wedge x, \theta_{200 x}=\alpha_{2} \wedge x, \theta_{102 x}=\beta_{1} \wedge x,
$$

where $\wedge$ is the minimum sign. Hence,

$$
\begin{aligned}
& E \theta_{221}=E \beta_{2}, E \theta_{210}=\int_{0}^{\infty} \bar{F}_{1}(t) \bar{F}_{2}(t) d t, E \theta_{112 x}=\int_{0}^{x} \bar{F}_{1}(t) d t, E \theta_{200 x}=\int_{0}^{x} \bar{F}_{2}(t) d t \\
& E \theta_{102 x}=\int_{0}^{x} \bar{G}_{1}(t) d t
\end{aligned}
$$

Using the formulas (1) let us find the average stationary time to failure TF $T_{+}$and the average stationary renewal time RT $T_{-}$.

Using the formulas (4) and (5), let us define the expressions comprised into the formulas

$$
\int_{E_{+}} \rho(d x) P\left(x, E_{-}\right)=\rho(210) P\left(210, E_{-}\right)+\int_{0}^{\infty} \rho(112 x) P\left(112 x, E_{-}\right) d x=\rho_{0} \int_{0}^{\infty} \hat{H}_{1}(y) f_{2}(y) d y,
$$


where $\hat{H}_{1}(y)=\sum_{n=0}^{\infty} F_{1}^{*(n)}(y)$ is the renewal function,

$$
\begin{gathered}
\int_{E_{+}} m(x) \rho(d x)=\rho(210) m(210)+\int_{0}^{\infty} \rho(112 x) m(112 x) d x= \\
=\rho_{0} \int_{0}^{\infty} d F_{2}(y) \int_{0}^{y} \bar{H}_{1}(y-t) \bar{F}_{1}(t) d t=\rho_{0} \int_{0}^{\infty} y d F_{2}(y)=\rho_{0} E \alpha_{2}, \\
\int_{E_{-}} \rho(d x) P\left(x, E_{+}\right)=\rho(221) P\left(221, E_{+}\right)+\int_{0}^{\infty} \rho(102 x) P\left(102 x, E_{+}\right) d x==\rho_{0} \int_{0}^{\infty} \bar{H}_{1}(y) d F_{2}(y) . \\
\int_{E_{-}} m(x) \rho(d x)=\rho(221) m(221)+\int_{0}^{\infty} \rho(102 x) m(102 x) d x=\rho_{0}\left(\int_{0}^{\infty} \bar{G}_{2}(y) d y+\right. \\
\left.+\int_{0}^{\infty} d x \int_{0}^{\infty} h_{1}(y) d_{y} F_{2}(x+y) \int_{0}^{x} \bar{G}_{1}(t) d t\right)=\rho_{0}\left(E \beta_{2}+\int_{0}^{\infty} \bar{G}_{1}(t) d t \int_{0}^{\infty} h_{1}(y) \bar{F}_{2}(t+y) d y\right) .
\end{gathered}
$$

Thus, the average stationary TF $T_{+}$, the average stationary RT $T_{-}$and the stationary availability factor $K_{a}$, of the system considered are approximately calculated by the formulas:

$$
\begin{gathered}
T_{+} \approx \frac{E \alpha_{2}}{\int_{0}^{\infty} \hat{H}_{1}(y) d F_{2}(y)}, T_{-} \approx \frac{E \beta_{2}+\int_{0}^{\infty} \bar{G}_{1}(t) d t \int_{0}^{\infty} h_{1}(y) \bar{F}_{2}(t+y) d y}{\int_{0}^{\infty} \widehat{H}_{1}(y) d F_{2}(y)}, \\
K_{a} \approx \frac{E \alpha_{2}}{E \alpha_{2}+E \beta_{2}+\int_{0}^{\infty} \bar{G}_{1}(t) d t \int_{0}^{\infty} h_{1}(y) \bar{F}_{2}(t+y) d y} .
\end{gathered}
$$

Provided the maintenance is carried out through the constant time interval $\tau \geq 0$ and taking into account that $F_{2}(t)=1(t-\tau)$ let us obtain the formula for $K_{a}$.

Hence,

$$
K_{a}(\tau) \approx \frac{\tau}{E \beta_{2}+\tau+\int_{0}^{\tau} H_{1}(\tau-t) \bar{G}_{1}(t) d t}
$$

Expression (8) can be used for solving the problem of finding the optimum value of the period $\tau$ of maintenance carrying out at which $K_{a}$ reaches the greatest value.

An example of finding of optimum value $\tau_{\text {onm }}$ of maintenance carrying out period using formula (8) with initial data [13] is represented in Table 1. 
Table 1. Optimal maintenance periodicity $\tau_{o p t}$ and correspondent values of $K_{a}\left(\tau_{o p t}\right)$

\begin{tabular}{|l|c|c|c||c|c||}
\hline \multicolumn{2}{|c|}{ Initial data } & \multicolumn{2}{c||}{ Result } \\
\hline \hline \multicolumn{1}{|c|}{ Kind of distribution } & $E \alpha_{1}, h$ & $E \beta_{1}, h$ & $E \beta_{2}, h$ & $\tau_{\text {opt }}, h$ & $K_{a}\left(\tau_{\text {opt }}\right)$ \\
\hline \hline 1. Exponential & 56 & 30 & 5 & $\infty$ & 0.651 \\
\hline 2. Erlangian of the $4^{\text {th }}$ order & 56 & 30 & 5 & 34 & 0.828 \\
\hline 3. Erlangian of the $6^{\text {th }}$ order & 56 & 30 & 5 & 36 & 0.846 \\
\hline 4. Erlangian of the $4^{\text {th }}$ order & 56 & 30 & 8 & 40 & 0.775 \\
\hline 5. Erlangian of the $4^{\text {th }}$ order & 50 & 30 & 5 & 31 & 0.815 \\
\hline
\end{tabular}

\section{Conclusion}

A lot of systems for various purposes allows the construction of a semi-Markov model. When constructing a semi-Markov model, it is necessary to build a rather complex phase space of states what impedes the finding of system's characteristics. In this case one can use the phase merging algorithms for an approximate finding of system's characteristics

In present paper, a semi-Markov model of the system was constructed with account for calendar maintenance. Approximate formulas were obtained for the stationary characteristics of the system using the algorithm of phase merging. For the case when the interval between consecutive maintenances is a constant value, the optimization of the function $K_{a} .(\tau)$ was carried out by the criterion of maximum.

In the future, this approach is supposed to be used to analyze the functioning of multicomponent technical systems taking into account maintenance.

The research was carried out within the state assignment of the Minobrnauki of Russia (No. 1.10513.2018/11.12), with financial support by RFBR (project No. 18-01-00392a).

\section{References}

1. V.A. Kashtanov, Semi-Markov Models of the Maintenance Process (Znanie, Moscow, 1987)

2. V.A. Kashtanov, A.I. Medvedev, Reliability Theory of the Composite Systems (Theory and Practice) (Fizmatlit, Moscow, 2010)

3. G.L. Curry, R.M. Feldman, Manufacturing Systems Modeling and Analysis, 2nd ed. (Springer-Verlag, Berlin Heidelberg, 2011)

4. F. Beichelt, P. Tittmann, Reliability and Maintenance: Networks and System (Taylor \& Francis Group: CRC Press, 2012)

5. J. MacGregor Smith, B. Tan (Eds.), Handbook of Stochastic Models and Analysis of Manufacturing System Operations (Springer-Verlag, New York, 2013)

6. V.A. Kashtanov, Optimum Problems of Maintenance (Znanie, Moscow, 1981)

7. F. Grabski, Semi-Markov Processes: Applications in System Reliability and Maintenance (Elsevier Science, 2014)

8. J. Jansen, N. Limnios (Eds.), Semi-Markov Models and Applications (Academic Publishers, Netherlands: Kluwer, 1999)

9. A.N. Korlat, V.N. Kuznetsov, M.M. Novikov, A.F. Turbin, Semi-Markov Models of Recoverable Systems and Queuing Systems (Shtiintsa, Kishinev, 1991) 
10. V.S. Korolyuk, A.F. Turbin, Markovian Restoration Processes in the Problems of System Reliability (Naukova Dumka, Kiev, 1982)

11. V.S. Korolyuk, Stochastic System Models (Lybid, Kiev, 1993)

12. V.S. Korolyuk, N. Limnios, Stochastic Systems in Merging Phase Space (World Scientific, Imperial College Press, 2005)

13. B.A. Kozlov, I.A.Ushakov, Handbook on the calculation of reliability of radioelectronics and automation equipment (Sovetskoye Radio, Moscow, 1975) 